\title{
EVIDENCIAS ARQUEOLÓGICAS DEL USO DEL BARRO COMO MATERIA PRIMA EN LA ARQUITECTURA ARGENTINA DEL PERIODO COLONIAL TEMPRANO
}

DOI: https://doi.org/10.18861/ania.2019.9.2898

Dra. Arq. Ana Igareta

ORCID iD: http://orcid.org/0000-0003-2510-794X 


\section{DRA. ARQ. ANA IGARETA}

Doctora en Ciencias Naturales, Universidad Nacional de La Plata (Argentina). Licenciada en Antropología, Universidad Nacional de La Plata (Argentina).

FECHA DE RECEPCIÓN: 31 de marzo de 2019.

FECHA DE ACEPTACIÓN: 19 de mayo de 2019.

REGISTRO BIBLIOGRÁFICO: IGARETA, A. (2019). Evidencias arqueológicas del uso del barro como materia prima en la arquitectura argentina del periodo colonial temprano. Anales de Investigación en Arquitectura, 9 (1), 7-24. 


\section{RESUMEN}

La fundación de ciudades en el norte y oeste de la República Argentina durante el primer siglo de conquista ibérica fue un complejo proceso de prueba, error y articulación de recursos y técnicas diversos. Las materias primas y sistemas constructivos utilizados en la arquitectura de tales ciudades continúa siendo uno de los grandes interrogantes de la arqueología colonial, dado que la mala conservación del registro construido limitó su análisis y desplazó el interés de los arqueólogos hacia otras evidencias, más abundantes y disponibles.

Un relevamiento sistemático de escritos de individuos que transitaron por la región durante el siglo XVI permitió detectar un acotado pero interesante conjunto de menciones referidas al uso de barro en la construcción de estructuras, dato que resultó consistente con lo observado en el terreno en intervenciones arqueológicas realizadas en las áreas fundacionales de algunas de estas ciudades. Dicho resultado abre la puerta a una caracterización más detallada de la arquitectura temprana de la región, hoy completamente desaparecida.

Palabras clave: arqueología colonial, materiales constructivos, barro.

\section{ABSTRACT}

The founding of cities in the north and west of the República Argentina during the first century of Iberian conquest was a complex process of trial, error and articulation of a variety of resources and techniques. Raw materials and constructive systems used such cities continue to be one of the great questions of colonial archeology, since the poor conservation of the architectural record limited its analysis and displaced archaeologists interests towards other evidences, more abundant and available.

A systematic survey of documents produced by individuals who traveled through the region during the sixteenth century allowed to detect a limited but interesting set of mentions referring to the use of raw clay in the construction of structures, consistent with archaeological finding in the foundational areas of some of those cities. It is expected that this result will contribute to the development of a more detailed characterization of its early architecture, now disappeared.

Keywords: colonial archaeology; building materials; raw clay. 


\section{CONSIDERACIONES GENERALES}

El proceso de conquista y colonización iniciado a fines del siglo XV desde la península ibérica sobre territorio americano tuvo extensas implicancias a nivel social en ambos continentes que quedaron reflejadas en el registro material. En la República Argentina, el surgimiento y consolidación de una red urbana articulada en los territorios de las provincias del norte y oeste del país ha sido, desde hace tiempo, tema de interés de investigaciones desarrolladas en el campo de la historia y la arquitectura (Gutiérrez, 1979; 1983). En las últimas décadas, la consolidación de proyectos integrales de arqueología colonial permitió avanzar desde el estudio de la evidencia material en la comprensión de los procesos de ocupación de esos nuevos espacios urbanos (Chiavazza y Ceruti, 2010), desde aspectos tales como el estudio de las nuevas relaciones sociales que se establecieron entre los grupos étnicos que convivieron en las ciudades, la caracterización del corpus de objetos, plantas y animales que circularon por la región y los circuitos de producción y circulación de bienes implicados en la consolidación de una dinámica novedosa, entre muchos otros.

Sin embargo, fueron contados los trabajos arqueológicos que se ocuparon desde la evidencia material de indagar en los rasgos físicos que tuvieron esas ciudades, o que se interesaron por relevar y sistematizar las características de su arquitectura, materias primas o sistemas constructivos empleados durante sus primeras décadas de existencia. Entre los pocos análisis estrictamente arqueológicos publicados al respecto cabe mencionar el de Bárcena y Schávelzon (1991) en el que revisan la información disponible sobre la construcción del Cabildo de Mendoza; el de Chiavazza y Anzorena (2006) de los materiales constructivos del predio de mercedarios del área fundacional de la misma ciudad y el de Spengler et al (2011) en el que presentan un estudio comparativo de la composición de adobes hallados en sitios arqueológicos formativos, incas y coloniales de La Rioja y Catamarca.

En el marco de un proyecto más amplio orientado a la caracterización de la materialidad de las ciudades coloniales tempranas del norte y oeste argentino, el objetivo del presente trabajo fue relevar los materiales y sistemas constructivos utilizados durante sus primeras décadas de existencia en una muestra de las instalaciones fundadas en la región durante el siglo XVI. Se buscó registrar información relativa a materias primas, modos de procesamiento y técnicas que aparecieran mencionados en fuentes documentales tempranas, y articularla luego con la evidencia material hallada en los sitios de dichas ciudades por los equipos arqueológicos que trabajaron en ellos. 


\section{CIUDADES COLONIALES TEMPRANAS}

Diversos relevamientos históricos han coincidido en estimar que cerca de veinte ciudades fueron fundadas durante el siglo XVI en las provincias que hoy conforman el noroeste argentino (Razori, 1945; Hardoy, 1972). En al menos cinco de esos casos, la fundación parece haber sido un evento meramente nominal y sin correlato material, y en otros seis que se trató de la relocalización en un sitio diferente de una población preexistente (Igareta 2008) por lo que en sentido administrativo no pueden ser consideradas como nuevas ciudades pero sus restos sí son susceptibles de ser identificados en el terreno. Así, más allá de la escasa superficie ocupada por la instalación o de la mínima cantidad de vecinos que tuvieron inicialmente, es posible proponer que las ciudades coloniales que llegaron a ocupar un lugar discreto en el espacio fueron El Barco I (1550), El Barco II (1551), El Barco III (1552), Santiago del Estero del Nuevo Maestrazgo (1553), Londres de la Nueva Inglaterra (1558), Córdoba de Calchaquí (1559), Cañete (1560), Mendoza del Nuevo Valle de la Rioja (1561), San Juan de la Frontera (1562), San Miguel de Tucumán y Nueva Tierra de Promisión (1565), Nuestra Señora de Talavera (1566), Lerma en el Valle de Salta (1582), Todos los Santos de la Nueva Rioja (1591), San Salvador de Velasco en el valle de Jujuy (1593) y San Luis de Loyola Nueva Medina de Rio Seco (1594).

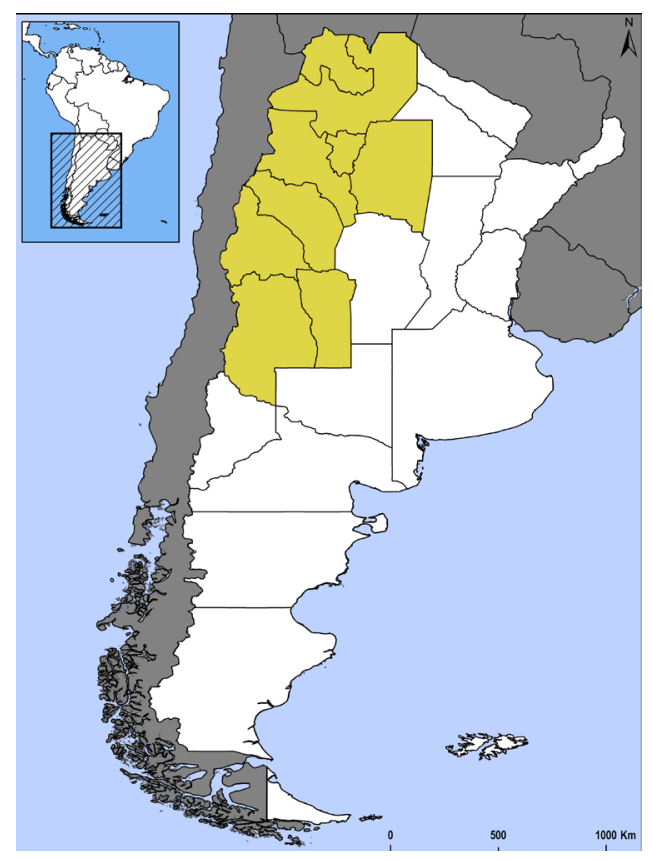

Figura $1 \mathrm{~A}$

Las provincias del norte y oeste de la República Argentina en las que se consolidó la primera red urbana del país en el siglo XVI.

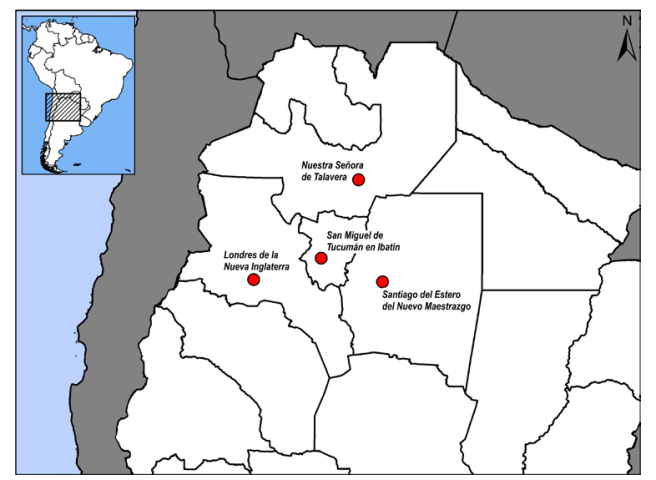

Figura 1B

Detalle con la ubicación de los sitios arqueológicos de las antiguas ciudades de Talavera, Tucumán, Londres y Santiago. 
No obstante, solo han sido identificadas arqueológicamente las áreas fundacionales de cinco de ellas : Santiago (Igareta, 2012), Londres (Igareta, 2008), Mendoza (Schávelzon, 2002), Tucumán (Gramajo de Martínez Moreno, 1976) y Talavera (Tomasini y Alonso 2001). Y si bien en los cinco sitios se produjo el hallazgo de un extenso registro mueble que contribuyó a la identificación, caracterización y asignación cronológica de su ocupación, el grado de conservación de los restos construidos en cada instalación es extremadamente variable, por lo que resultó difícil realizar comparaciones entre los conjuntos o detectar elementos en común.

Dejando momentáneamente de lado el registro arqueológico de la ciudad de Mendoza y concentrándonos en las cuatro instalaciones ubicadas más al norte, los restos arquitectónicos tempranos detectado por los arqueólogos en Santiago y Talavera se reducen a montículos longitudinales de tierra de escasa potencia que apenas se diferencian del sedimento del suelo que los rodea (Igareta, 2012:259). Pero mientras que en Santiago solo se han hallado unas pocas secciones conservadas de menos de un metro de largo y no fue posible identificar a que sector de la primitiva ciudad podía corresponder la estructura a la que pertenecían, en las ruinas de Talavera los montículos son más numerosos y más extensos, y algunos convergen formando ángulos rectos (Tomasini et al, 2004:608), lo que hizo posible elaborar un plano tentativo de su temprana traza urbana. En lo que respecta a solados, en ambos sitios se hallaron restos de pisos de tierra apisonada asociados a los montículos, lo que contribuyó a su identificación de éstos como la base de antiguos muros de adobe o tapial, pero su grado de deterioro impidió identificar si habían sido erigidos utilizando uno u otro sistema constructivo. En Tucumán, en cambio, se hallaron montículos semejantes asociados a cimientos de piedra sin cantear, pese a que también en este caso la sección de tierra de los muros ha desaparecido casi por completo y tampoco pudo determinarse con que sistema constructivo fueron realizados (Rivet,2008:94). Pero la buena conservación de los cimientos líticos permitió identificar la planta de numerosas construcciones del área central de la ciudad, en las inmediaciones de la plaza e identificar con precisión a que edificio mencionado en los documentos históricos correspondía cada una de las principales estructuras (Gramajo de Martínez Moreno, 1976; Eugenio, 2000). En el sitio de Londres, por su parte, el registro arquitectónico del periodo colonial temprano incluye dos grandes edificios con muros perimetrales de piedra canteada con mínimos porcentajes de mortero de barro divididos en siete recintos menores mediante tabiques de tapial en cuya manufactura se utilizaron piedras canteadas procedentes de otras estructuras a modo de material de relleno y abundante barro (Igareta, 2008; Figura 2). El acotado tiempo de ocupación del sitio (menos de cinco años) no parece haber permitido que alguno de los recintos adquiriera funciones específicas, y tampoco se observaron diferencias en tal sentido en el 
Figura 2A

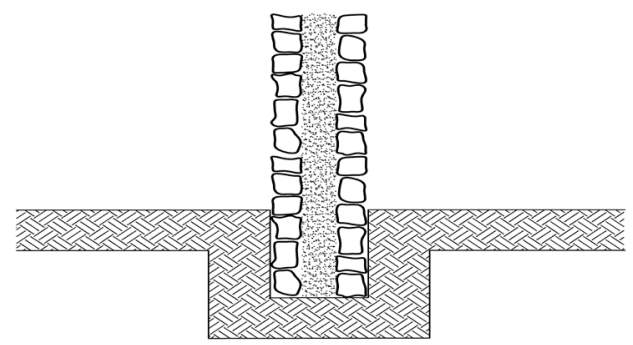

Figura 2B

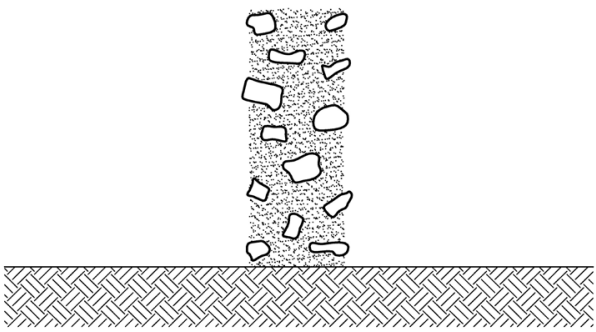

Corte esquemático que muestra los dos sistemas constructivos identificados arqueológicamente en el sitio fundacional de Londres:

Figura 2A

Muro perimetral doble de piedra canteada y escaso mortero intersticial con relleno barro (edificado durante el periodo inca)

Figura 2B

tabique divisorio de tapia con inclusiones de bloques de piedra canteada de mediano tamaño colocadas desordenadamente construido durante la temprana ocupación colonial; nótese el detalle de que los tabiques históricos fueron construidos sin cimientos.

registro material in situ. Tanto en Londres como en Tucumán se hallaron en estratigrafía restos de vigas de madera que formaron parte de los techos, evidencia de su derrumbe cuando las estructura aún se hallaban en uso, mientras que no se dispone de datos arqueológicos sobre techumbres para Santiago o Talavera.

\section{El rastreo de información histórica que permitiera} referenciar el variado registro material hallado en los sitios de Santiago, Londres, Tucumán y Talavera a través de descripciones generales de las ciudades, de cualquiera de sus edificios o de algún aspecto puntual de su materialidad en momentos tempranos, no arrojó resultados relevantes. Como bien señala Lafaille Gentile, las noticias sobre los territorios ubicados al sur de Charcas durante el siglo XVI suelen ser breves, esporádicas, parciales, sesgadas y acotadas
(Lafaille Gentile, 2014). Así, a diferencia de lo que ocurre con poblaciones contemporáneas de Chile o Perú, no fue posible hallar documentos públicos o privados producidos durante el siglo XVI por individuos que transitaron por la región que describieran o caracterizaran el aspecto físico de sus ciudades.

Síabundan, en cambio, descripciones regionales en las que se nombra a las ciudades; se detalla la distancia que separa a una de otra y el estado de los caminos; se brindan rasgos particulares sobre su geografía, ubicación y recursos; se mencionan los beneficios o malestares que su clima genera en la salud de sus habitantes y los motivos de conflicto entre ellos y con las parcialidades indígenas. Pero con frecuencia, ni siquiera es posible identificar a que centro urbano en particular se están refiriendo las 
menciones, dado que los relatos son confusos o los autores presentan caracterizaciones generales a escala regional. Ocasionalmente los documentos incluyen menciones específicas a capillas o iglesias de una u otra orden, pero generalmente para indicar si contaban o no con cantidad suficiente de religiosos, aunque en algunos casos también dan cuenta del buen o mal estado de esos edificios.

Descripciones como la que sigue, referida a la ciudad de Talavera, son recurrentes en los relatos de funcionarios y cronistas de la época:

“... edificada á la ribera de un rio grande que en veranos solo se vadea. Los vecinos estaban descontentos del asiento, porque la madre del rio es arenisca y no pueden hacer molinos en él, y como dicen se han mudado casi 25 leguas hacia Salta, a un asiento mucho mejor del mismo temple y más fresco, llamado Palca Tucumán donde se pueden sacar acequias y hacer molinos y para pacificar a unos indios belicosos llamados Lules."

(Lizárraga, [1589] 1916: 232).

Una primera revisión documental tampoco permitió detectar representaciones gráficas de ninguna de las cuatro ciudades de la muestra, de algunos de los edificios construidos en ellas o del aspecto general de su traza realizadas durante el siglo XVI por individuos que las hayan observado en persona. Cabe mencionar que sí se conocen dibujos y planos de algunas de estas ciudades pero en todos los casos realizadas por individuos que nunca transitaron por la región, lo que obliga a un análisis crítico de su potencial informativo. Buen ejemplo de ello es la representación de una Tucumán insular incluida en el conocido texto de Guamán Poma de Ayala (Guamán Poma de Ayala, [1615] 1987: 1155). Puede especularse que el cronista peruano, que nunca visitó la ciudad, escuchó relatos de terceros en los que se hablaban de las frecuentes inundaciones que la afectaban y estimó por ello que se trataba de una isla y así la dibujó, pero la posibilidad solo reafirma la importancia de trabajar con documentos producidos por individuos que efectivamente hayan visitado los sitios en cuestión (sus relatos deben ser considerados igualmente de modo crítico para identificar potenciales distorsiones, voluntarias o involuntarias, pero cuentan con el beneficio de basarse en una materialidad observada, sensu Lorandi y del Rio, 1992). Tampoco se conocen planos post facto de ninguna de las ciudades creadas en el noroeste argentino durante el siglo XVI y producidos en el mismo periodo que brinden datos que permitan referenciarlas geográficamente (Hardoy, 1991), y ya ha sido discutida la escasa representatividad de los planos fundaciones generados a priori de la existencia material de la ciudad (Igareta, 2018a). 


\section{NUEVA PROPUESTA DE ANÁLISIS}

En este punto de la investigación, y teniendo en cuenta que los datos documentales registrados no habían podido ser integrados de modo efectivo con la evidencia material identificada en el terreno, se propuso una nueva revisión de fuentes escritas pero desde una perspectiva diferente: buscando identificar referencias a las materias primas y/o sistemas constructivos. Aceptando la ausencia de descripciones articuladas en textos del siglo XVI referidos a la arquitectura de las ciudades consideradas, se propuso la hipótesis de que los cronistas podrían, en cambio, haber hecho referencia a ciertos aspectos puntuales de la misma, y que la integración de esa información con el registro hallado en los sitios contribuiría a la comprensión de la historia constructiva del conjunto.

Para la nueva revisión se seleccionó una muestra de fuentes de primera mano, documentos producidos entre mediados del siglo XVI y la primera década del siglo XVII por individuos que transitaron las ciudades de Santiago, Londres, Tucumán y Talavera. Los elegidos fueron los textos incluidos en "Gobernación del Tucumán: Papeles de gobernadores en el siglo XVI", recopilación y transcripción de Levillier (1920); la “Descripción colonial" de Lizárraga ([1589] 1916); el "Interrogatorio para las Indias Occidentales de 1604 y los informes remitidos por el teniente de gobernador, vecinos, moradores y residentes de Nuestra Señora de Talavera en 1608", transcripción de Simioli et al (2017) y la "Relación de las provincias del Tucumán" de Pedro Sotelo de Narváez 1582, transcripción de Lafaille Gentile (2012). Se buscó relevar todas las menciones directas detectadas referidas a materiales de construcción, técnicas de procesamiento y preparación de materias primas, sistemas constructivos y dificultades halladas en cualquiera de las categorías antes mencionadas. De igual modo se relevaron - por separado- todos aquellos datos considerados como referencias indirectas a alguna de dichas categorías, como por ejemplo las reiteradas menciones al deterioro que las lluvias producían en los muros de algunos edificios aunque no se detallara el material de dichos muros, por estimarse que la información podía resultar relevante a posteriori al articularla con la proporcionada por otras fuentes.

Por tratarse de una primera aproximación arqueológica a la problemática propuesta, no se pretendió realizar una evaluación del grado de confiabilidad de cada documento o del relato de cada autor, sino generar una base de datos general cuyo contenido pudiera ser luego sujeto a evaluación crítica y contrastación. De igual modo, en esta instancia no se buscó identificar -aunque se registró en caso de que fuera detallado- a que edificio en particular hacían referencia las menciones, limitándonos a relevar la asociación propuesta entre un determinado 
Figura 3

Corte esquemático de un muro que muestra los dos sistemas constructivos de tierra cruda que podrían haber sido usados en Santiago y Talavera durante el periodo colonial temprano. .

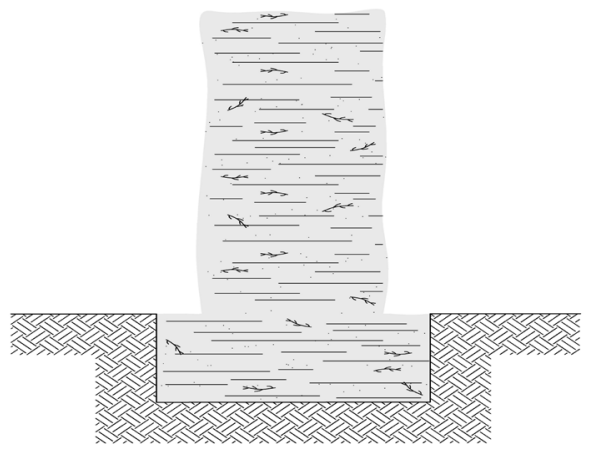

Figura 3a. Tapial

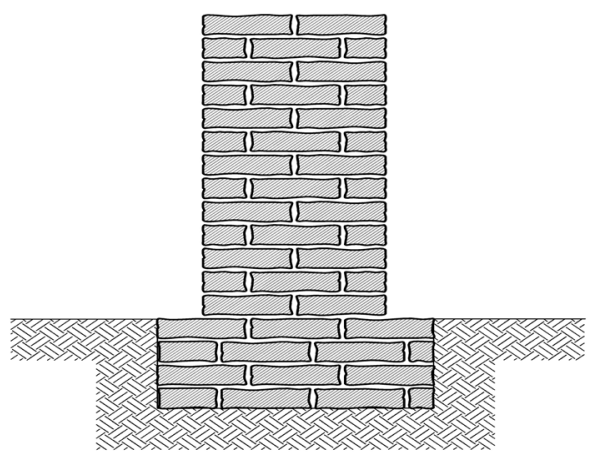

Figura 3b. Adobes

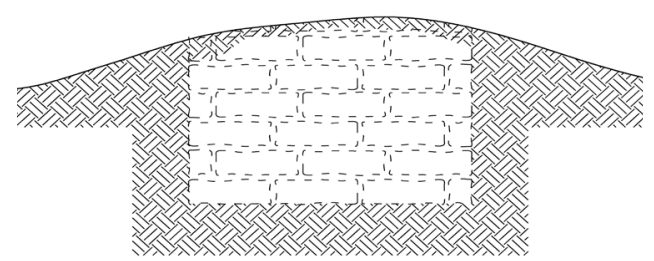

Figura 3c. Corte esquemático que representa los restos hallados en las excavaciones realizadas en el sito cuya erosión y desgaste hicieron imposible diferenciar el sistema empleado para su construcción. material o técnica en un determinado sitio durante el periodo considerado. La información proporcionada por los textos históricos fue articulada con los datos publicados referidos al registro material presente en cada sitio con el objetivo de identificar coincidencias y recurrencias que no hubieran sido detectadas con anterioridad y que sirvieran para proponer nuevas posibilidades de análisis.

Este intento de reconocer y atribuir un conjunto de características específicas a la estructuras de un periodo particular se realizó con pleno reconocimiento de que la arquitectura es un hecho dinámico, en permanente transformación, tanto en su configuración y usos del espacio como en su materialidad (Rapoport, 1972), pero aceptando que análisis acotados de este tipo son una herramienta válida para el estudio de ciertos aspectos de la misma.

\section{RESULTADOS Y DISCUSIÓN}

La nueva revisión documental permitió identificar un limitado pero bien definido conjunto de referencias a diversos aspectos de la construcción de edificios en momentos tempranos, y detectar coincidencias significativas entre las fuentes. A nuestro entender, una de las más interesantes fue la mención a la edificación de viviendas con tierra cruda: el relato de testigos y cronistas señala la utilización alternativa o combinada de adobes y de tapial como sistema constructivo en tres de las cuatro ciudades, no habiéndose 
hallado mención alguna a Londres. "El edificio de las casas [en Santiago] es de adobes, como en las demás ciudades..." (Lizárraga, [1589] 1916:112). "Las casas [en Talavera] son echas de adobes y tapias y la cubierta de terrado y de la misma manera son las mas de las ciudades de la Governaçion (...) Y las çercas de las casas son de tapias de tierra..." (Diego López de Correa, 1608 en Simioli et al, 2017:13). El dato resulta consistente con lo observado en el terreno en las áreas fundacionales de Santiago y Talavera donde el barro parece haber sido el único material empleado en los paramentos, dado que no se han hallado restos de piedra ni siquiera en los cimientos de ninguno de los edificios tempranos (Igareta, 2012; Tomasini et al, 2004) cuyo registro se limita a montículos lineales acotados (Figura 3). En Tucumán, en cambio, se observó el uso de piedra bola (rodados naturales sin cantear recuperados de los cursos de los ríos) en los cimientos de los principales edificios cívicos y religiosos del sitio, estimándose que sobre ellos se erigieron paredes de barro que han desaparecido en su totalidad. En otros sectores del antiguo casco urbano de esta ciudad cuya superficie no fue aún intervenida ni se realizaron excavaciones enfocadas en exponer los cimientos de piedra, se ha observado la presencia de montículos cubiertos por vegetación que podrían corresponder a antiguos muros, pero no se han desarrollado hasta ahora acciones sistemáticas destinadas a liberarlos, por lo que tampoco en este caso se dispone de información arqueológica que indique si el sistema
Figura 4

Corte esquemático que muestra los dos sistemas constructivos de tierra cruda que podrían haber sido usados para el desarrollo de muros sobre cimientos líticos en la Tucumán colonial:

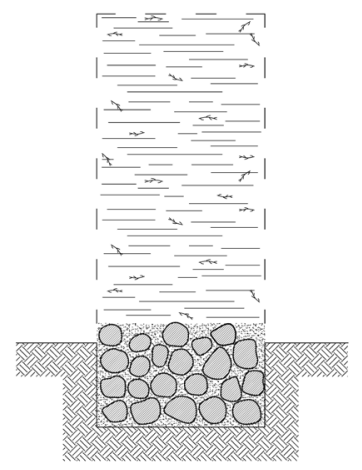

Figura 4a. Tapial

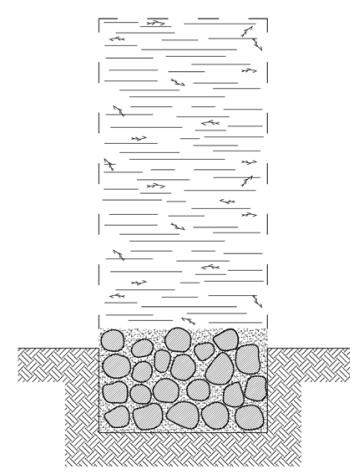

Figura 4b. Adobes

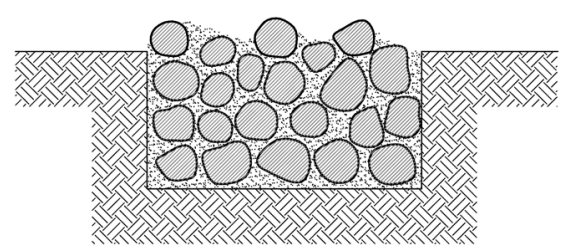

Figura 4c

Corte esquemático que muestra la única evidencia de construcciones hasta ahora identificada en el sitio: cimientos de piedra bola unidas con mortero de barro. 


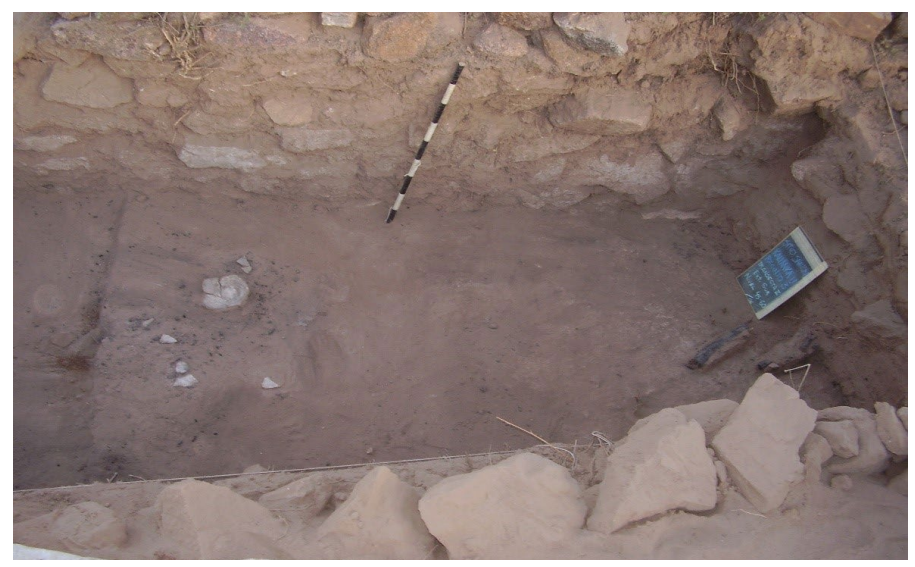

Figura 5.

Vista del interior de uno de los recintos incas reocupados durante la fundación de Londres; a la derecha, se observan dos secciones de vigas que pertenecieron al techo construido durante el periodo colonial.

empleado fue el tapial o superposición de adobes. (Figura 4). La solidez que los cimientos de piedra dio originalmente a la arquitectura de esta ciudad podría explicar por qué aparece señalada con frecuencia como la “... de mejores edificios...” (Lizárraga, [1589] 1916:112) de todo el territorio.

Igualmente interesantes resultaron las referencias históricas a los materiales usados en los techos de las estructuras, sobre todo si se tiene en cuenta que no se han encontrado hasta hoy restos de techos en su posición original o aún articulados con otros elementos constructivos en ningún sitio colonial temprano de la Argentina. Por ejemplo, el hallazgo de restos de tirantes quemados y con clavos de hierro de sección cuadrada que constituyen la única evidencia material de techumbre hallados en Londres (Igareta, 2008:224, Figura5), coincide con una mención referida al uso de ciertas especies como materia prima para construcción en el territorio del Tucumán: “... saca madera de çedros e nogales para todos los pueblos de la tierra [de la Gobernación] porque es muy abundante della ..." (Sotelo de Narváez 1582 en Lafaille Gentile, 2012: 603). Resulta interesante señalar que se trata de la única mención registrada en los documentos revisados en la que se hace referencia a madera y no a leña, dado que referencias a esta última sí abundan en los textos, y a nuestro entender supone dos usos completamente diferentes para el material vegetal (solo el primero relacionado directamente a construcción).

Pero sin duda el dato más interesante en relación a este componente fueron las reiteradas menciones al uso de barro como materia prima de la cobertura de los techos, aunque ninguna de ellas la atribuyera a una ciudad en particular. Simplemente se indica 
para las poblaciones tempranas que “... las casas si no es cual o cual; cúbrenlas con unos terrados de mas de una tercia de grueso, muy bien pisados con pisones..." (Lizárraga [1589] 1916:108). El dato resulta significativo para el análisis de dicho componente en las ciudades de la muestra (particularmente en momentos tempranos, previos al inicio de la producción de tejas) y un indicio orientador para futuras búsquedas en el terreno, dado que solo se conoce un único hallazgo en tal sentido. En el sitio de Tucumán se detectó, durante la excavación de una vivienda particular, una capa de sedimento ceniciento con gran cantidad de pequeños carbones que correspondería a la quemazón de la cubierta de paja de su techo (Rivet 2008:98) .

Las repetidas menciones a los efectos negativos que la salinidad de los suelos tenía sobre las edificaciones de Santiago y Talavera pueden ser consideras también como evidencia indirecta del uso de tierra cruda y otros componentes orgánicos como principal material constructivo en ambas ciudades y contribuir a explicar por qué su registro arquitectónico es tan exiguo 500 años después.. “... en estas dos [Santiago y Talavera], como la tierra es salitrosa, vase desmoronando el adobe y cada año es necesario reparar las paredes..." (Lizárraga [1589] 1916:112). “...por estar aquí [en Santiago] poblados en vn salitral que cada quatro años es menester hazer las casas (...) a mi me paresce conuiene mudalla para la perpetuidad y bien de los naturales (...) y a mi parecer a menester otro tanto la ciudad de nuestra señora de talayera porque esta fundada en otro salitral y con los mesmos inconvenientes ..." (Ramírez de Velazco, 1590 en Levillier, 1920:288).

La falta de piedra en los territorios de Santiago y de Talavera y la friabilidad de los suelos es un dato que los documentos mencionan repetidamente para dar cuenta de las dificultades halladas por sus pobladores al momento de intentar la construcción de estructuras de mayor durabilidad que las de barro.

“Los vecinos estaban descontentos del asiento [de Talavera] porque la madre del rio es arenisca y no pueden hacer molinos en él..." (Lizárraga, [1589] 1916:110).

“... padecese en esta ciudad [Santiago] por no haber molino ni poderse hacer porque como ya dijimos estos reinos ser de esa calidad; pasan por tierra arenisca, donde no se halla una piedra, ni se puede hacer ni sacar acequia dellos, a la primera venida, allá va todo" (Lizárraga [1589] 1916:112).

“... no tiene rremedio de sacar acequias [en Santiago] por auer ahondado el rrio y ser la tierra muy llana y arenisca y no tener fixeza en toma que se haga ni auer genero de piedra en munchas leguas y no poder auer molino..." (Ramírez de Velazco, 1590 en Levillier, 1920:278). 


\section{CONSIDERACIONES FINALES}

Los resultados obtenidos en el marco del presente análisis constituyen un pequeño primer paso en lo que se propone ser un abordaje sistemático del estudio arqueológico de las características de la arquitectura doméstica del noroeste argentino durante el periodo colonial temprano. La concurrencia observada entre las fuentes con respecto al uso barro como materia prima-aunque con diferentes aplicaciones es el único material presente en todos los sitios- fue un resultado alentador en tal sentido que debe ser ampliado. Persiste el interrogante acerca de qué sistema constructivo fue utilizado en cada caso, ya que además del adobe y tapial nombrados en los documentos, los incipientes hallazgos realizados en otros sitios de la región de cronología semejante (Chiavazza y Anzorena, 2006; Igareta, 2018b) obligan a considerar la posibilidad de que al menos parte de las construcciones hayan sido edificadas con quincha, un sistema basado en el uso de postes de madera, ramas y barro cuyo registro material podría ser semejante al de los materiales antes mencionados. En tal sentido, muestras de barro obtenidas de los restos de muros hallados en los cuatro sitios están siendo analizadas con el objetivo de caracterizar su contenido botánicos, a fin de utilizar dicha información para la interpretación de la cadena operativa implicada en la preparación de diferentes mezclas (Castillón et al, 2018) y en el procesamiento y uso diferencial de cada material (Igareta y Castillón, 2018).
Este tipo de análisis, susceptible de brindar información sobre los procesos y sistemas constructivos empleados en los sitios incluso cuando la mayor parte de las estructuras ha desaparecido y solo se conservan escasos elementos de las mismas resulta una alternativa interesante para el estudio de un componente del registro arqueológico hasta ahora relegado. Cabe esperar que la articulación progresiva de información novedosa proporcionada por nuevas excavaciones y por nuevas lecturas de textos históricos permita caracterizar con mayor detalle los materiales y técnicas utilizadas en los edificios de esas primeras ciudades, y avanzar luego en la indagación de aspectos relacionados con quiénes fueron los actores involucrados en los procesos constructivos, qué tradiciones entraron en juego en los mismos, qué rasgos singulares surgieron a nivel local o cuales fueron motivos que llevaron a la utilización de ciertos recursos por sobre otros. 


\section{BIBLIOGRAFÍA}

BARCENA, R. \& SCHÁVELZON, D. (1991). El Cabildo de Mendoza. Arqueología e Historia para su recuperación. Mendoza: Municipalidad de Mendoza.

CASTILLÓN, V.; LÓPEZ, M. L. \& IGARETA, A. (2018). Arquitectura y plantas. Análisis de microrrestos botánicos en argamasas en el sitio arqueológico El Shincal (Catamarca, Argentina) durante los siglos XV y XVI. En: Alcántara, M.; García Montero, M. \& F. Sánchez López (coordinadores), Memoria del $56^{\circ}$ Congreso Internacional de Americanistas. Ciencias y Medio Ambiente. Vol. 4 (pp. 234247). Salamanca: Ediciones Universidad de Salamanca.

CHIAVAZZA, H. \& ANZORENA, J. (2006). Estudio de materiales constructivos obtenidos en las excavaciones arqueológicas del predio de los mercedarios. En: Chiavazza, $\mathrm{H}$ y Zorrilla, V. (editores), Arqueología en el predio mercedario de la ciudad de Mendoza (pp. 211-262). Mendoza: Facultad de Filosofía y Letras, Universidad Nacional de Cuyo.

CHIAVAZZA, H. \& CERUTI, C. (editores). (2010). Arqueología de ciudades americanas del siglo XVI. Mendoza: Facultad de Filosofía y Letras, Universidad Nacional de Cuyo.

EUGENIO, E. (2002). “Investigaciones arqueológicas en Tucumán, antigua San Miguel de Tucumán". Actas del Primer Congreso Nacional de Arqueología Histórica. Ed. Corregidor. Buenos Aires.

GRAMAJO DE MARTÍNEZ MORENO, A. (1976). La primitiva ciudad de San Miguel de Tucumán en Tucumán. Estudio histórico-arqueológico. Relaciones de la Sociedad Argentina de Antropología. Vol. X, N.S (pp.141-165).

\section{GUTIÉRREZ, R.}

(1979). Arquitectura colonial: teoría y praxis. Buenos Aires: Instituto Argentino de Investigaciones en la Historia de la Arquitectura y el Urbanismo

(1983). Las propuestas morfológicas del urbanismo hispánico. En: Morresi, E. y Gutiérrez, R. (Dir.), Presencia Hispánica en la Arqueología Argentina, Vol. 1 (pp. 4564). Resistencia: Instituto de Historia de la Universidad Nacional del Nordeste.

\section{HARDOY, J.}

(1972). Las formas urbanas europeas durante Ios siglos XVI y XVII y su utilización en América Latina. En: Urbanización y proceso social en América. Lima: IEP.

(1991). Cartografía urbana colonial de América Latina y el Caribe. Instituto Internacional de Medio Ambiente y Desarrollo - América Latina. Buenos Aires: Grupo Editor Latinoamericano.

IGARETA, A.

(2008). Últimas noticias desde Londres - EI sitio histórico más antiguo de la arqueología de Catamarca. (Tesis de doctorado inédita). Universidad Nacional de La Plata. Facultad de Ciencias Naturales y Museo. La Plata.

(2012). Arqueología de Santiago del Estero colonial: historia de varias ciudades. En: Jorge Buján (comp.). Ciudades y territorio en América del Sur - Del siglo XV al XVII (pp. 235 a 263). La Plata: Editorial Nobuko.

(2018a, octubre). Quincha tal vez - Dificultades en la identificación de cimientos de quincha 
en el registro colonial de San Juan. Ponencia presentada en el VII Congreso Nacional de Arqueología Histórica Argentina. Facultad de Humanidades y Artes, Universidad Nacional de Rosario, Rosario.

(2018b) San Juan de la Frontera en el siglo $\mathrm{XVI}$ : fragmentos de su imagen fundacional. Andinas, Revista de Estudios Culturales, Facultad de Arquitectura, Urbanismo y Diseño de la Universidad Nacional de San Juan. Año 7, n॰6(pp. 6-15).

IGARETA, A. \& CASTILLÓN, V. (2018, julio). Materias primas y sistemas constructivos en la arquitectura doméstica de Argentina durante el periodo colonial temprano: "Amenísimas regiones que también tienen su dragón...". Ponencia presentada en el $56^{\circ}$ Congreso Internacional de Americanistas, Salamanca.

2007. IGARETA, A. Y GONZÁLEZ LENS, D. Ocupación post-incaica en El Shincal de Quimivil: nuevas evidencias arquitectónicas. Memorias del Tercer Congreso de Historia de Catamarca, Tomo I (pp. 135-150). Catamarca: Junta de Estudios Históricos de Catamarca.

\section{LAFAILLE GENTILE, M.}

(2012). Geografía y política. La gobernación de Tucumán en 1582, según la Relación de Pedro Sotelo Narváez. Anuario Jurídico y Económico Escurialense 45 (pp. 581-608).

(2014). La gobernación de Tucumán c. 1570 - Comentarios a la "Relación de los pueblos descubiertos por Gerónimo Luis de Cabrera, gobernador de los Juríes". Revista Cruz de Sur. Año IV, núm. 6 (pp. 11-84).
LEVILLIER, R. (1920). Gobernación del Tucumán: Papeles de gobernadores en el siglo XVI. Madrid: Imprenta J. Pueyo.

LIZÁRRAGA, R. ([1589] 1916). Descripción Colonial. Libro primero, Colección Biblioteca Argentina. Buenos Aires: Librería La Facultad de Juan Roldán.

LORANDI, A.M. \& DEL RÍO, M. (1992). La etnohistoria - Etnogénesis y transformaciones sociales andinas. Buenos Aires: Centro Editor de América Latina.

RAZORI, A. (1945). Historia de la ciudad argentina. Tomo I. Buenos Aires: Imprenta López.

RIVET, C.

(2008). Pasado y presente de una ciudad colonial: El caso Tucumàn (1565-1685). Arqueología histórica en un espacio doméstico. (Tesis de licenciatura inédita). Universidad Nacional de Tucumán. Facultad de Ciencias Naturales Instituto Miguel Lillo. San Miguel de Tucumán.

(2010). El espacio doméstico en la primera San Miguel de Tucumán, un caso de estudio arqueológico. En: Chiavazza, H. \& C. Ceruti (editores). Arqueología de ciudades americanas del siglo XVI (pp. 165-202). Mendoza: Facultad de Filosofía y Letras, Universidad Nacional de Cuyo.

SCHÁVELZON, D. (editor). (2002). El Área Fundacional de Mendoza. Mendoza: Centro de Investigaciones Ruinas de San Francisco y Centro de Arqueología Urbana, FADU, UBA. 


\section{FUENTE DE LAS IMÁGENES}

SIMIOLI, J., PORTERIE, A. \& MARSCHOFF, M. (2017). El Interrogatorio para las Indias Occidentales de 1604 y los informes remitidos por el teniente de gobernador, vecinos, moradores y residentes de Nuestra Señora de Talavera en 1608. Presentación y transcripción completa. Corpus Archivos virtuales de la alteridad americana,Volumen 7, $N^{\circ} 1$. Consultado en diciembre de 2018. https://journals.openedition.org/ corpusarchivos/1888

SPENGLER, G.; DO CAMPO, M. \& RATTO, N (2011). Caracterización de materiales constructivos en tierra mediante estudios de laboratorio. En: S. Bertolino, R. Cattáneo y A. Izeta (eds.). La arqueometría y la arqueología en Latinoamérica (pp. 309-320). Córdoba: Universidad Nacional de Córdoba. Facultad de Filosofía y Humanidades.

TOMASINI, A.\&ALONSO, R. (2001). Esteco el Viejo. Breve historia de Nuestra Señora de Talavera (1566-1609). Salta: Góficalmpersora.

TOMASINI, A.; CURZIO, D. \& VIVEROS, F. (2004) Arqueología de Nuestra Señora de Talavera (1566-1609). XXIV Encuentro de Geohistoria Regional (pp.607-614). Resistencia: Instituto de Investigaciones Geohistóricas CONICET.
Figura 01: a) y b) Elaboración propia de Diego Gobbo (División Arqueología, Museo de La Plata, UNLP) a partir de datos SIG vectoriales de la CONAE (2019).

Figura 02: a) y b) Dibujos Arq. Florencia Chechi (Equipo Arqueología Histórica, Museo de La Plata, UNLP)

Figura 03: a), b) y c) Dibujos Arq. Florencia Chechi (Equipo Arqueología Histórica, Museo de La Plata, UNLP)

Figura 04: a), b) y c) Dibujos Arq. Florencia Chechi (Equipo Arqueología Histórica, Museo de La Plata, UNLP)

Figura 05: Fotografía de la autora. 
24 Anales de Investigación en Arquitectura Vol.9 | 2019 | № 1. Montevideo (Uruguay), 7-24 Universidad ORT Uruguay 